\title{
$\mathbf{m} / \mathbf{S} 2000$
}

médecine/sciences 2000 ; $16: 1207-10$

\section{Les enjeux de santé publique dans les pays développés}

\section{Pierre Lombrail Thierry Lang}

P. Lombrail : Laboratoire de santé publique et d'épidémiologie, Faculté de médecine, 1, rue Gaston-Veil, 44035 Nantes Cedex, France. T. Lang : Département d'épidémiologie et santé publique, Faculté de médecine, 37, allées de Guesde, 31000 Toulouse, France.
Les enjeux de santé publique dans les pays occidentaux sont multiples. Ils tiennent autant aux transformation prévisibles de nos sociétés qu'à la difficulté de gérer un système de soins toujours plus complexe. Pour que ces enjeux soient posés au mieux, il est nécessaire que la santé publique acquière droit de cité dans le débat public.

\section{TIRÉS À PART}

P. Lombrail.

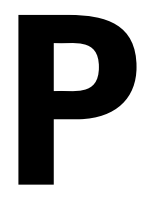

rétendre traiter des enjeux de santé publique dans l'ensemble des pays dits développés dans une note aussi brève relève de la gageure. Nous nous appuierons en grande partie sur l'exemple français pour cette raison. Dans son premier rapport sur la santé en France [1], le Haut Comité de la Santé Publique (HCSP) dressait un constat d'ensemble favorable tout en faisant état de "faiblesses structurelles» et de "menaces de détérioration ». II mettait en exergue le poids particulier des maladies chroniques, pour partie rançon de l'allongement de l'espérance de vie, et celui des maladies liées aux comportements à risque (au premier rang desquels le tabagisme et la consommation excessive d'alcool); il soulignait la montée du mal-être psychologique et social ; il attirait l'attention sur les conditions de vie des personnes en situation de handicap ou de dépendance et les inégalités, notamment sociales, de santé. Les enjeux ainsi esquissés concernent en premier lieu le système de santé; au-delà, ils soulèvent une question majeure, celle des liens entre santé et société, qu'une somme de travaux empiriques ne permet plus de considérer comme hypothétiques. Nous aborderons ainsi les enjeux pour le système de santé, que posent le vieillissement démographique, l'exclusion sociale et la participation des citoyens au système de soins, puis les enjeux posés par les déterminants sociétaux.

\section{Le système de soins}

\section{Le vieillissement des sociétés} occidentales

Nous ne détaillerons pas les conséquences de la double transition démographique et épidémiologique abordée dans ce numéro. Nous ne ferons qu'en souligner une conséquence, la prévalence croissante des maladies chroniques en évoquant certaines de ses implications. La première est le défi lancé à une médecine encore trop sûre de prouesses techniques dans le registre biomédical dont bénéficient quelques-uns, quand c'est en termes de préservation du capital d'autonomie de tous que le vieillissement de nos sociétés oblige à penser par une approche globale, "bio-psycho-sociale», des problèmes. Si l'objectif n'est plus « seulement » de rajouter des années à la vie mais aussi de la vie aux années, il devient incontournable de permettre aux personnes concernées d'exprimer leurs attentes et leurs perceptions (au mieux, de susciter leur formulation) puisqu'elles seules sont à même de dire ce qu'elles estiment être leur qualité de vie. Un défi supplémentaire vient de l'évidence que ni la recherche de l'autonomie ni celle de la qualité de vie ne peuvent se résu- 
mer au seul résultat des soins puisque I'une comme l'autre sont fortement dépendantes de nos conditions de vie. Au minimum, cette recherche suppose que des professionnels d'horizons divers, du sanitaire et du social, parviennent à coordonner leur action, éventuellement par la création de « réseaux de soins».

\section{Précarité sociale et inégalités sociales de santé et de soins}

Les inégalités face à la santé, loin de se résorber, s'accroissent en France [2] comme ailleurs, et les pays de l'ex-Union soviétique en donnent un exemple frappant. Conséquences de la question sociale [3], elles se traduisent par une surmortalité et une surmorbidité évitables des catégories sociales les moins favorisées [1] qui révèlent pour partie les dysfonctionnements de notre système de soins [4]. Un quart de nos concitoyens déclarent avoir dû renoncer à des soins au moins une fois dans leur vie [5]. Si les inégalités de droit sont en passe d'être fortement atténuées sinon résolues avec I'avènement d'une couverture maladie universelle, nombre de barrières demeurent, qu'elles soient financières, cognitives ou culturelles [6].

Ces inégalités sociales recoupent pour partie aussi une stratification sociale de certains comportements "individuels» à risque, et de la consommation excessive d'alcool en premier lieu. Prévenir ou limiter les effets délétères de ces comportements relève en partie du domaine de l'éducation pour la santé et soulève des questions éthiques difficiles en rapport avec le risque de contrôle social. Pour F. Bertolotto, "I'enjeu n'est pas seulement de "soigner" un corps, mais bien plus de (re)donner à un sujet les conditions de sa santé (...), et de l'aider à être bien dans l'environnement qui est à l'origine de son "mal-être", sans pour autant jouer sur la plupart des déterminants qui ont provoqué cet état» [7]. C'est dire la difficulté de cette approche. Intégrer la dimension sociale des comportements, prendre en compte le fait qu'ils sont à la croisée de l'individuel et du social est un défi que doit relever l'activité des professionnels de (I'éducation pour) la santé [8].

La précarisation de la situation sociale d'une frange croissante de nos sociétés engendre une souffrance psychique considérable. Cette souffrance questionne violemment les modèles de I'intervention psychiatrique dans notre pays. Certains professionnels, soucieux de ne pas outrepasser leurs missions, refusent toute implication hors de situations strictement pathologiques et identifiées comme telles, d'autres sont plus interventionnistes mais sur un registre dominant biomédical. Dans les deux cas, la réponse n'est pas satisfaisante [9]. Cette remise en question du soin psychiatrique par la fracture sociale, n'est que la manifestation la plus évidente de la mauvaise gestion de la santé mentale de l'ensemble de la population dont la forte consommation de psychotropes dans notre pays est un symptôme parmi d'autres. Ce symptôme est une traduction de la médicalisation de la "peine à vivre» d'un nombre croissant de personnes dont les repères se brouillent dans un univers qui valorise à outrance une certaine forme de "performance» individuelle au détriment du lien social, soulignant dès lors les limites de cette intervention [10].

\section{La difficile gestion de l'innovation technologique}

Nous ne prendrons que deux exemples: les progrès de la génétique et I'extension des domaines d'intervention du médicament. Les progrès spectaculaires de la génétique ouvrent des perspectives cliniques révolutionnaires et agitent de ce fait des intérêts financiers considérables. Il faut souhaiter qu'un véritable débat sur I'utilisation de ces connaissances ait lieu. Au-delà de résultats thérapeutiques indéniables, le développement des techniques génétiques de dépistage est-il porteur de discriminations, vis-à-vis du travail, de l'assurance face à la maladie, voire des soins? Comment éviter l'effet d'enfermement dans un groupe à risque, à la suite d'une annonce de test qui peut transformer une vie?

L'innovation pharmaceutique bouscule les frontières du normal et du pathologique et questionne les limites de la médicamentation d'attentes relevant de la conformité à une représentation sociale dominante du bienêtre, éventuellement en dehors de tout recours au système de soins et de ses possibilités de contrôle des effets adverses pour la santé. Dans son avis rendu le 6 avril 2000 sur « la médicalisation de la sexualité: le cas du Viagra $^{\circledR}$ » et donc sur le remboursement par la collectivité de la prescription de silfenadil, notre Comité Consultatif National d'Éthique propose de définir les limites entre traitement de troubles organiques et intervention " de confort » visant à pallier certaines défaillances de la sexualité masculine. Ces défaillances relèvent d'une "interaction complexe de facteurs physiques et mentaux» dont la complémentarité "peut signifier au plan thérapeutique que I'on dépasse la simple prise de médicament». C'est pointer $d^{\prime}$ 'une autre manière la tentation de médicaliser certains troubles qui naissent $d^{\prime}$ un décalage entre les capacités individuelles et la valorisation médiatique d'une certaine vision de la «performance».

\section{Les déficiences du système de santé}

Dans son diagnostic initial, le Haut Comité de la Santé Publique pointait en France une série de limites d'un système qu'il qualifiait de figé : "insuffisance de pilotage et de régulation», déséquilibres multiples (suprématie de l'hôpital sur la ville, du "soin» (curatif) sur la prévention, qu'elle soit primaire ou tertiaire, cloisonnements à tous les niveaux et «manque d'évaluation». Ce constat particulièrement accusé dans un système $d^{\prime}$ inspiration libérale se retrouve à des degrés divers dans l'ensemble des pays occidentaux.

Ce dernier point souligne un défi majeur à nos yeux, qui consisterait à remettre les professionnels de soin dans le jeu de la régulation. Formés avant tout à la prise en charge individuelle de maladies caractérisées suivant une rationalité biomédicale, ils doivent investir une approche collective de problèmes complexes qui n'ont souvent de «solution » que dans une approche globale faisant intervenir des acteurs complémentaires. Pour ce faire, ils doivent investir le champ de l'organisation pour être à même de garantir continuité et coordination des soins. Instruits dans une logique d'obligation de moyens, ils découvrent une obligation de résultat de plus en plus pressante. Au-delà de la dimension juridique de cette muta- 
tion (difficile gestion de la responsabilité sans faute), c'est le regard du malade-usager qui s'insinue également dans une relation soignant-soigné encore volontiers paternaliste. Car si c'est ce dernier qui peut juger de la nature du service rendu, c'est lui surtout dont la coopération devient indispensable à l'obtention du meilleur résultat possible. Enfin, l'imputabilité problématique d'un résultat final à une intervention en particulier entraîne une responsabilité partagée qui devrait rendre solidaires I'ensemble des parties concernées. Ce qui justifie d'autant plus la nécessité d'innovations organisationnelles comme la constitution de réseaux de soins ou le développement de pratiques d'évaluation collective.

L'avènement du malade-usager précède ou accompagne celle du malade-citoyen. Celui qui réclame le droit à la santé mais qui exige également les moyens de gérer sa propre santé. C'est ouvrir le chantier de ce que nos amis canadiens appellent l'empouvoierement des malades ou de chacun d'entre nous. Ce qui devrait conduire à donner la place qui lui revient à la promotion de la santé telle que définie par l'Organisation Mondiale de la Santé en 1986 dans la charte d'Ottawa: «La promotion de la santé vise l'égalité en matière de santé. Ses interventions ont pour but de réduire les écarts actuels caractérisant l'état de santé, et d'offrir à tous les individus les mêmes ressources et possibilités pour réaliser leur potentiel santé. Celà comprend une solide formation dans un milieu apportant son soutien, I'information, l'aptitude et les possibilités de faire des choix sains. Les gens ne peuvent réaliser leur potentiel de santé optimal s'ils ne prennent pas en charge les éléments qui déterminent leur état de santé.»

\section{Enjeux sociétaux: au-delà du système de soins, faire de la santé un objet de débat public}

On sait peu de choses sur la nature des risques et les effets de la dégradation accélérée de notre environnement naturel, et le peu qui est su est très mal partagé, quand il n'est pas occulté. Ce n'est qu'en 1997 qu'un programme de recherche européen sur les maladies liées à la pollution (de l'air) a été financé [11]. On ne connaît clairement ni la nature ni la force du lien avec la dégradation des trois éléments essentiels à la vie que sont I'air, l'eau potable et les sols et I'augmentation de l'incidence de certains cancers, de l'asthme et de certaines allergies. Une véritable gestion du risque environnemental supposerait une stratégie rigoureuse associant l'identification des dangers potentiels et la mesure de l'exposition humaine à ces dangers. En l'absence de certitudes, le principe de précaution devrait imposer des actions préventives. Ce dossier aussi souligne la nécessité d'un abord global des politiques de santé. Maîtriser le risque environnemental ne peut être qu'un élément d'une politique d'ensemble visant la protection de l'écosystème, la prévention de la surexploitation des ressources naturelles comme celle du réchauffement de la planète. Cela supposerait une prise de conscience collective de notre responsabilité vis-à-vis des générations futures [12].

Nos sociétés portent une attention faible aux risques professionnels, à leur prévention et à leur indemnisation [13]. L'absence de visibilité sociale des effets du travail peut expliquer en partie cette situation tout comme la réticence de certains employeurs de voir reconnaître I'entièreté d'un risque dont ils supportent le coût de l'indemnisation en France. La part croissante des effets à long terme, nécessitant un suivi prolongé au-delà de la vie professionnelle, les modifications du travail (chômage, sous-traitance et autres conséquences de la précarisation des emplois les plus exposés) qui en compliquent le suivi sanitaire rendent cette entreprise difficile mais ne sauraient servir d'alibi à l'inaction.

Les inégalités sociales et géographiques de santé posent de façon fondamentale cette question. Elles ne sont pas seulement cette précarité visible qui interroge le système de santé, elles sont aussi des écarts croissants d'état de santé qui suivent la "hiérarchie» sociale, des manœuvres aux cadres supérieurs. Ces inégalités ne relèvent qu'en partie seulement de l'accès aux soins ou des comportements dits «à risque». Dans un ouvrage collectif américain du nord [14], un récent rap- port de I'OMS [15] ou le rapport remis par Sir Acheson au gouvernement anglais [16], les soins n'apparaissent qu'au milieu d'un ensemble de déterminants de santé, aujourd'hui solidement étayés par des travaux scientifiques de qualité. L'habitat, la zone de résidence, les transports, les liens sociaux (support et réseaux sociaux), les contraintes du travail, l'emploi ou son absence, déterminent la santé et la force du lien n'est en rien marginale par rapport à des variables biomédicales établies.

Un enjeu majeur de la santé publique est sans doute $d^{\prime}$ intégrer la santé comme un des critères de jugement pour décider l'opportunité d'une politique publique quelconque ou pour en évaluer les effets. La dimension européenne n'est pas tant un enjeu qu'une évidence. Pour ne prendre qu'un exemple, la santé publique, à la différence des prix du marché, n'est pas un critère des politiques agricoles communes. Pourtant, la distribution des excédents de produits laitiers dans les écoles a des effets sanitaires, la subvention de la culture du tabac en Europe n'en est certainement pas dépourvue.

\section{Conclusions}

Au terme de ce survol, un enjeu qui paraît peut-être le plus problématique consisterait à maîtriser le processus d'émergence et de définition de ce qui peut être considéré comme un enjeu de santé publique. Cet enjeu a au moins deux facettes: I'une est politique et l'autre est technique.

Sur un plan politique, sachant la rationalité limitée des experts, qui s'accordent difficilement entre eux, comme le montre encore un récent travail de spécialistes de santé publique européens [17], la question de la "démocratie sanitaire» se trouve posée. Sommes-nous en mesure de "renforcer l'action communautaire» au sens entendu par la charte d'Ottawa: "La promotion de la santé procède de la participation effective de la communauté à la fixation des priorités, à la prise de décision et à l'élaboration des stratégies de planification, pour atteindre un meilleur niveau de santé »?

Sur un plan technique, dans notre pays, le Haut Comité de la Santé Publique, après avoir rappelé par 
exemple les insuffisances caractérisant les conditions de vie des personnes en situation de handicap ou de dépendance, soulignait le déficit des connaissances en la matière: "l'épidémiologie reste à faire (...) et la recherche est extrêmement déficiente, qu'il s'agisse, entre autres, de la représentation sociale, de l'économie du handicap, ou du vieillissement des personnes handicapées » [1]. Un véritable débat sur la santé publique et ses priorités suppose un choix éclairé par des faits, issus de la surveillance et de la recherche en épidémiologie et en santé publique, qui restent à développer et consolider dans notre pays. Quantifier les risques, les comparer, les ordonner ne fait pas la décision; c'est pourtant une étape essentielle pour guider les choix au même titre qu'une évaluation bien conduite de l'impact des interventions en cours ou envisageables. Pourtant, à supposer que les "vrais enjeux» émergent, rien ne garantit qu'une prise en charge adaptée s'ensuive et les inégalités actuelles de santé et d'accès aux soins nous le rappellent durement [18]

\section{RÉFÉRENCES}

1. Haut Comité de la Santé Publique. La santé en France. Rapport général. Paris: La Documentation Française, 1994: 333 p.

2. Bihr A, Pfefferkorn R. Les inégalités face à la santé. In: Déchiffrer les inégalités. Paris : Syros, 1999: 229-53.

3. Castel R. Les métamorphoses de la question sociale, une chronique du salariat. Paris: Fayard, 1995.
4. Chauvin P, Lebas J. La problématique précarité et santé, un symptôme des dysfonctionnements de notre système de santé. In: Lebas J, Chauvin P, eds. Précarité et santé. Paris: Flammarion, 1998: 3-22.

5. Dumesnil S, Grandfils N, Grignon M, Le Fur P, Ordonneau C, Sermet C. Santé, soins et protection sociale en 1997. Paris: CREDES, 1999; $167 p$.

6. Lombrail P. Accès aux soins. In : Leclerc A, Fassin D, Grandjean H, Kaminski M, Lang $\mathrm{T}$, eds. Inégalités et disparités sociales en santé. Paris: La Découverte, 2000 (sous presse)

7. Bertolotto F. L'éducation pour la santé face à la «fracture sociale». L'éducation pour la santé. Du discours à la pratique. Actualité et Dossiers en Santé Publique 1996; 16: XXIX-XXXI.

8. Brixi $\mathrm{O}$, Lang $\mathrm{T}$. Comportements et inégalités de santé. Inégalités sociales de santé. In: Leclerc A, Fassin D, Grandjean H, Kaminski M, Lang T, eds. Inégalités et disparités sociales en santé. Paris: La Découverte, 2000 (sous presse).

9. Minard M, Piel E. La prise en charge des exclus. In: Kovess V, Lopez A, Pénochet JC, Reynaud M, eds. Psychiatrie années 2000. Paris: Flammarion Médecine-Sciences, 1999: 99-105.

10. Jaeger $M$. La médicalisation psychiatrique de "la peine à vivre». In: L'ère de la medicalisation. Sous la direction de Pierre Aïach et Daniel Delanoë. Paris: Anthropos, 1998: 135-58.

11. Brinch B, Carlsen A, Fabricius A, et al. Environment. In: Brodin M, Weil O, McKee M, Oberlé D, eds. Priorities for public health action in the European union. Bruxelles: Commission européenne, mars 1999: 122-37.

12. Jonas H. Pour une éthique du futur. Paris: Payot, 1998.

13. Cassou B. Un constat inquiétant. Tribune libre dans le dossier Santé et Travail. Actualité et dossier en santé publique. Haut Comité de la Santé Publique 1994; 9 : XXIXXXXV.

14. Evans RG, Barer ML, Marmor TR (sous la direction de). Être ou ne pas être en bonne santé. Biologie et déterminants sociaux de la maladie. Paris: John Libbey Eurotext, 1996; $359 \mathrm{p}$.

15. WHO Regional Office for Europe. Social determinants of health. The solid facts. Copenhague: OMS, 1998.

16. Acheson D. Independant inquiry into inequalities in health. Report. Londres: The Stationery Office, 1998.

17. Oberlé D, Weil O, McKee M, Brodin M. Des professionnels de santé publique européens pour exprimer dix priorités pour 'Union européenne... à suivre. Santé publique $1999 ; 11$ : 409-25.

18. Leclerc A, Fassin D, Grandjean $H$, Kaminski M, Lang T. Inégalités et disparités sociales en santé. Paris: La Découverte, 2000 (sous presse).

\section{$\mathbf{m} / \mathbf{s} 2000$}

\section{Summary}

Public health issues

in the Western world

Public health issues encompass the health care system itself and the society as a whole. The health care system must face several changesor issues: ageing and persisting inequalities in health and access to health care, the difficult management of new technologies and of the health care system itself. But this professional approach must be challenged by a democratic one where public health enters into the public arena. 